\title{
Fluoridation of drinking water reduces caries prevalence
}

\author{
McDonagh M, Whiting P, Bradley M, Cooper J, Sutton A, Chestnutt I, et al. A Systematic Review of Public Water \\ Fluoridation, September 2000. York: University of York Publications Office, NHS Centre for Reviews and Dissemination. \\ ISBN 1900640163
}

Objective To determine the effects of fluoridation of drinking water supplies on the incidence of caries.

Data sources See page 37.

Study selection See page 37.

Data extraction and synthesis See page 37.

Results Twenty-six studies met the criteria. There were no level 'A' studies. All but three were before and after studies, two used prospective cohort designs, and one a retrospective cohort. The most serious defect of these studies was the lack of appropriate analysis. The degree of caries reduction is not clear from the data available. It was estimated that a median of six people need to receive fluoridated water for one extra person to be caries-free [interquartile range of study number-needed-to-treat (NNT), 49]. Studies after withdrawal of water fluoridation (level 'B' and limited quantity) indicate that caries prevalence increased, approaching the level of the low fluoride group. The estimates of effect could be biased due to poor adjustment for the effects of potential confounding factors.
Table 1 Difference between fluoridated and non-fluoridated water

\begin{tabular}{lcc}
\hline Mean & Range & Median (interquartile range) \\
\hline Caries-free children (\%) & $-5.0-64 \%$ & $14.6 \%(0.05-22.1 \%)$ \\
Change in dmft/DMFT score & $0.5-4.4$ & 2.25 teeth (1.28-3.63 teeth) \\
\hline dmft, primary decayed, missing and filled teeth; DMFT, permanent \\
decayed, missing and filled teeth.
\end{tabular}

Conclusions The best available evidence suggests that fluoridation of drinking-water supplies does reduce caries prevalence, both as measured by the proportion of children who are caries-free and by the mean change in $\mathrm{dmft} / \mathrm{DMFT}$ score. The studies were of moderate quality (level B), but of limited quantity.
Evidence-Based Dentistry
(2002)
3, 39. doi:10.1038/sj. ebd.6400101

Address for reprints: Publications Section, NHS Centre for Reviews and Dissemination, University of York, Heslington, York YO10 5DD, UK.

\section{Commentary}

This systematic evaluation of public water fluoridation provides a welldesigned review and a comprehensive approach to data sources. Every effort was made to ensure that all relevant literature was searched. Valid criteria for study selection were used. In addition, utmost attention was given to data extraction and synthesis, and to summarising the results and conclusions.

With the high level of interest and oftencontentious public debate regarding public water fluoridation, it is surprising that little high-quality research has been done. Few studies have been designed to assess the effects of water fluoridation over time. It is notable that, although this review presents a summary of the best available and most reliable evidence on the safety and efficacy of water fluoridation, the research evidence is of insufficient quality to allow confident statements about the evidence of a benefit in reduction in caries versus the increased prevalence of dental fluorosis. Likewise, the insufficient quality of evidence prevents confident conclusions about other potential harms, or whether there is an impact on social inequalities. The most serious deficiency in thesestudies was thelack of appropriate analysis of the data. Many studies did not include an analysis at all, whereas others carried out only simple analyses without attempting to control for potentially confounding factors.

The NNT represents the number of children who need to receive the intervention for one extra child to be cariesfree, with a $95 \%$ CI of between five and nine people. This NNT figure is a relatively accurate estimate based on the data from the studies included in this analysis.

The best available evidence suggests that fluoridation of drinking water supplies does in fact reduce caries prevalence, both when measured by the proportion of children who are caries-free, and by the mean change in dmft/DMFT score. However, to have real confidence in the ability to determine whether the fluoridation of drinking water reduces caries prevalence, the quality of evidence would need to be higher. The failure of these studies to deal with potential confounding factors or to provide standard error data means that the ability to definitively answer the question is limited. The studies were of moderate quality, but of limited quantity. Future research into the safety and efficacy of water fluoridation should be conducted with methodology that can improve the quality of the evidence base.

Richard D Bebermeyer

Department of Restorative Dentistry and Biomaterials,

The University of Texas Health

Science Center, Houston-Dental Branch, Houston, Texas, USA 